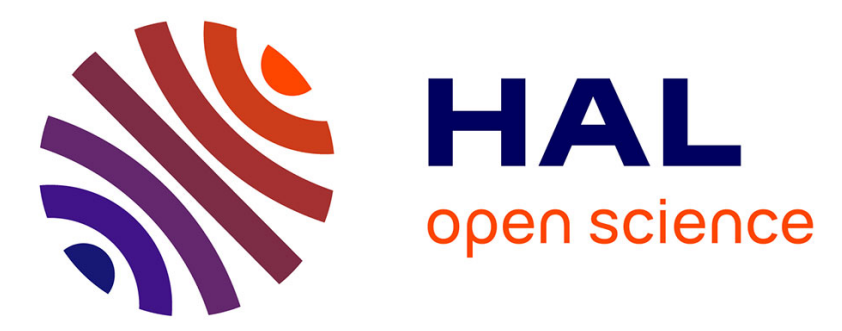

\title{
Libertés factuelles et contraintes fictionnelles: les descriptions américaines de Chateaubriand et leur mise en récit dans Atala, Les Natchez et le Voyage en Amérique

\author{
Alain Guyot
}

\section{To cite this version:}

Alain Guyot. Libertés factuelles et contraintes fictionnelles: les descriptions américaines de Chateaubriand et leur mise en récit dans Atala, Les Natchez et le Voyage en Amérique. Bulletin de l'association Guillaume Budé , 1996, 55 (4), pp.357-372. 10.3406/bude.1996.2410 . hal-02357822

\section{HAL Id: hal-02357822 \\ https://hal.science/hal-02357822}

Submitted on 10 Nov 2019

HAL is a multi-disciplinary open access archive for the deposit and dissemination of scientific research documents, whether they are published or not. The documents may come from teaching and research institutions in France or abroad, or from public or private research centers.
L'archive ouverte pluridisciplinaire HAL, est destinée au dépôt et à la diffusion de documents scientifiques de niveau recherche, publiés ou non, émanant des établissements d'enseignement et de recherche français ou étrangers, des laboratoires publics ou privés. 


\section{Libertés factuelles et contraintes fictionnelles : les descriptions} américaines de Chateaubriand et leur mise en récit dans Atala, Les Natchez et le Voyage en Amérique Alain Guyot

\section{Citer ce document / Cite this document :}

Guyot Alain. Libertés factuelles et contraintes fictionnelles : les descriptions américaines de Chateaubriand et leur mise en récit dans Atala, Les Natchez et le Voyage en Amérique. In: Bulletin de l'Association Guillaume Budé : Lettres d'humanité, n55, décembre 1996. pp. 357-372;

doi : https://doi.org/10.3406/bude.1996.2410

https://www.persee.fr/doc/bude_1247-6862_1996_num_55_4_2410

Fichier pdf généré le 11/05/2018 


\section{Libertés factuelles et contraintes fictionnelles : les descriptions américaines de Chateaubriand et leur mise en récit dans Atala, Les Natchez et le Voyage en Amérique}

L'authenticité du voyage de Chateaubriand en Amérique a fait l'objet de nombreuses études ${ }^{1}$, qui ont le plus souvent conclu au caractère absolument fictif de bien des trajets et des rencontres retracés par l'auteur dans l'ensemble de son œuvre, de l'Essai historique aux Mémoires ${ }^{2}$. C'est pourquoi, au lieu d'envisager la question des descriptions américaines sous l'angle d'une référentialité qui reste hypothétique, il peut s'avérer plus intéressant - et peut-être plus pertinent - d'étudier la manière dont cette matière descriptive s'intègre aux différents ouvrages de Chateaubriand. On sait qu'elle trouve son origine dans le fameux manuscrit pour partie composé "dans le désert, et sous les huttes des Sauvages ${ }^{3}$, dont l'existence ne nous est connue que par les témoignages de l'auteur lui-même : c'est de cette matière parfois informe, parfois beaucoup plus élaborée, que naîtront les descriptions de l'Amérique qui jalonnent l'Essai comme le Génie, Atala comme Les Natchez, le Voyage en Amérique comme les Mémoires, sans oublier les fréquentes allusions présentes dans l'Itinéraire ou le Voyage en Italie. Chateaubriand a certes le souci de ne pas offrir au lecteur la même description d'une œuvre à l'autre, ce dont

1. Cf. en particulier celle que R. Switzer a placée en tête de son édition du Voyage en Amérique (Paris, Didier, 1964).

2. Cela n'est d'ailleurs pas sans influer sur le contenu factuel et la forme de mainte description du récit de voyage, comme des œuvres réflexives ou autobiographiques : comment décrire des lieux que l'on n'a pas vus en laissant supposer qu'on les a vus ? Si le procédé n'est pas nouveau chez les voyageurs qui l'ont précédé, Chateaubriand y déploie tous les trésors d'un incomparable art de persuader : qu'on songe en particulier à la fameuse "Description de quelques sites dans l'intérieur des Florides " qui, dans le Voyage en Amérique, conclut la partie proprement consacrée à l'itinéraire.

3. Préface d'Atala dans OEuvres romanesques et voyages [M. Regard éd.], Paris, Gallimard, Pléiade, 1969, t. I, p. 18. Nos références à cet ouvrage, ainsi qu'aux Natchez et au Voyage en Amérique, seront toutes empruntées à cette édition. Pour l'histoire du manuscrit primitif, cf. l'introduction donnée par J.-Cl. BERCHET à son édition d'Atala. René. Les Natchez (Paris, L.G.F., 1989, p. 16 et suiv.). 
témoignent les nombreux renvois ${ }^{4}$. Mais il n'est pas rare qu'il manifeste avec brio son art du remploi : une confrontation de ces descriptions de même source - et de même référent - s'impose donc.

Encore faut-il s'aventurer hors des chemins largement battus par les exégètes de la "Nuit chez les sauvages du Nouveau Monde " 5 , et prendre garde aux pièges de la critique génétique. La chronologie de la composition des ouvrages tirés du manuscrit primitif reste en effet passablement obscure : de bien des pages d'Atala, des Natchez ou du Voyage en Amérique, écrites et le plus souvent remaniées, à des distances temporelles parfois vertigineuses à l'échelle d'une vie humaine, on ne saurait dire laquelle a la préséance sur l'autre. C'est pourquoi, à une mise en perspective génétique, on préférera une confrontation générique, au sens où l'on envisagera ces descriptions de référent semblable en fonction du genre littéraire auquel appartient l'ouvrage dans lequel elles s'insèrent : les différences que l'on trouve entre elles sont-elles significatives d'un point de vue stylistique ? Peut-on en tirer la matière d'un éventuel cahier des charges propre à chacun des genres dans lesquels s'inscrivent les ouvrages concernés? Quel usage Chateaubriand fait-il de ce cahier des charges? Du même coup, on éliminera d'emblée l'hypothèse qui ferait apparaître les descriptions du récit de voyage comme une sorte de " gamme de l'écriture ", de matière préparatoire à la fiction narrative. On aura donc affaire, dans ces confrontations descriptives, moins à des situations de récriture qu'à une sorte de dialogue en contrepoint masqué entre deux formes narratives autonomes, disposant de règles et de moyens propres. Afin d'éviter la dispersion dans une matière aussi complexe et un champ d'exploration aussi nouveau, on restreindra le propos à quelques exemples significatifs de descriptions mises en récit dans Atala, Les Natchez et le Voyage en Amérique.

On connaît l'hétérogénéité de ce dernier ouvrage, qui fut publié vingt-cinq ans après Atala : la première partie, consacrée au récit du périple à travers l'Amérique du Nord, se compose de textes rédigés au moment de la publication - soit trente-six ans après le voyage, de fragments tirés du manuscrit - ou supposés tels, et de commentaires contemporains de la révision, qui sont ccnsćs réunir ces fragments et, partant, les authentifier. La seconde consiste en une chorographie de la faune et de la flore de l'Amérique

4. L'auteur y fait allusion dans l'A vertissement du Voyage en Amérique (p. 615).

5. Que l'on songe aux multiples variations sur les variantes de ce passage, chez A. Albalat (Les corrections de Chateaubriand, dans Le Travail du style [...], Paris, 1903), V. Giraul) (Histoire des variations d'une page de Chateaubriand, dans Chateaubriand. Études littéraires, Paris, 1904), M. TISON-BRAUN (Poétique du paysage, Paris, 1980, p. 59 et suiv.), G. Grinette (Palimpsestes, Paris, 1982, p. 275 et suiv.). 
du Nord, suivie d'une étude sur les mœurs et les institutions politiques des Indiens. Le récit de voyage se termine sur une série de considérations politiques concernant l'état des Sauvages en 1827, ainsi que le développement des États-Unis et des Républiques espagnoles d'Amérique, suivies d'une conclusion et de notes complémentaires.

C'est dans la seconde partie que l'on retrouve le plus grand nombre de descriptions communes au récit de voyage et aux deux ouvrages de fiction. Cela n'est guère étonnant : on connaît la nature ethnographique avant la lettre du projet initial des Natchez ${ }^{6}$. Mais le caractère généralement didactique des descriptions du Voyage en Amérique, hérité du grand récit d'exploration cher à la seconde moitié du XVIII ${ }^{e}$ siècle, doit tenir compte des exigences propres à la fiction narrative ?

Du coup, les descriptions textuellement reprises d'une œuvre à l'autre, ou sujettes à d'infimes variations, ne sont pas légion. Dans leur immense majorité, celles du Voyage en Amérique sont d'une étendue bien supérieure à leurs homologues fictionnelles, le plus souvent réduites à quelques lignes ou intégrées qu'elles sont, comme en passant, au tissu narratif ${ }^{8}$. C'est probablement l'une des lois d'airain de la fiction romanesque qui se met en place dans ces premières années du XIX ${ }^{e}$ siècle : discours pressé, voire " contraint ", selon l'expression de $\mathrm{Ph}$. Hamon, elle privilégie l'action au détriment de la vision - on est encore bien loin des silences de Flaubert...

C'est ainsi que nombre de descriptions didactiques extrêmement précises du récit de voyage ne trouvent place dans les ouvrages de fiction narrative que si elles présentent un intérêt pittoresque ou dramatique. C'est le cas d'un développement à valeur réflexive ayant pour thème les mœurs des Indiens Iroquois : sur le modèle du jésuite Lafitau, Chateaubriand rapporte explicitement aux principes de Lycurgue l'éducation qui était donnée aux jeunes membres de cette tribu. Il soutient sa démonstration par une série d'illustrations en insistant sur les " jeux mâles et virils " auxquels s'adonnaient ces Spartiates du Nouveau Monde. L'un d'eux retient particulièrement son attention :

"Souvent de petits garçons s'attachaient les bras ensemble, met-

6. Cf. J-Cl. Bercher, ouvr. cité, p. 11.

7. Ce terme paraît le moins inadéquat pour désigner les objets complexes que sont, du point de vue des genres littéraires, Atala ou Les Natchez. Dans la suite, on utilisera l'adjectif fictionnel pour évoquer la fiction sous cet angle esthétique, et pour ne pas reprendre l'adjectif fictif: qui renvoie davantage à un horizon logique et véridictionnel.

8. La proportion avoisine les quatre-vingt-dix pour cent... 
taient un charbon ardent sur leurs bras liés, et luttaient à qui soutiendrait plus longtemps la douleur " (Voyage en Amérique, p. 850851).

Or cette notation est reprise à peu près textuellement dans le tableau du village des Natchez qui ouvre le roman homonyme:

" [...] de jeunes garçons, les bras attachés ensemble, s'essayaient à qui supporterait le plus longtemps l'ardeur d'un charbon enflammé " (p. 169).

Le cadre en est tout différent, puisque cette séquence se situe parmi d'autres descriptions d'actions parallèles présentant les activités auxquelles se livrent les Indiens à la tombée du soir. Dans ce contexte, le morceau se voit dépourvu de sa fonction d'exemplum, et ne semble plus se maintenir en régime fictionnel que pour exalter, par quelque trait marquant, le pittoresque exotique et un peu inquiétant des mœurs de ces tribus que le cinéma contribuera plus tard à populariser...

On aboutirait sans doute à une conclusion similaire en confrontant la fameuse description du conseil des Muscogulges, qui décide du sort réservé à Chactas dans Atala, et son homologue du Voyage en Amérique :

"Les conseils des nations indiennes se composent des chefs des tribus, des chefs militaires, des matrones, des orateurs, des prophètes ou jongleurs, des médecins ; mais ces conseils varient selon la constitution des peuples.

Le spectacle d'un conseil de Sauvages est très pittoresque. Quand la cérémonie du calumet est achevée, un orateur prend la parole. Les membres du conseil sont assis ou couchés à terre dans diverses attitudes : les uns, tout nus, n'ont pour s'envelopper qu'une peau de buffle; les autres, tatoués de la tête aux pieds, ressemblent à des statues égyptiennes; d'autres entremêlent, à des ornements sauvages, à des plumes, à des becs d'oiseau, à des griffes d'ours, à des os de castor, à des dents de poisson, entremêlent, dis-je, des ornements euro-
"Le conseil s'assemble. Cinquante vieillards, en manteau de castor, se rangent sur des espèces de gradins faisant face à la porte du pavillon. Le grand chef est assis au milieu d'eux, tenant à la main le calumet de paix à demi coloré pour la guerre. À la droite des vieillards, se placent cinquante femmes couvertes d'une robe de plumes de cygnes. Les chefs de guerre, le tomahawk à la main, le pennache en tête, les bras et la poitrine teints de sang, prennent la gauche.

Au pied de la colonne centrale, brûle le feu du conseil. Le premier jongleur, environné des huit gardiens du temple, vêtu de longs habits, et portant un hibou empaillé sur la tête, verse du baume de copalme sur la flamme et offre un sacrifice au solcil. Ce triple rang de vieillards, de ma- 
péens. Les visages sont bariolés de diverses couleurs, ou peinturés de blanc ou de noir. On écoute attentivement l'orateur; chacune de ses pauses est accueillie par le cri d'applaudissement, oah! oah!" (Voyage en Amérique, p. 832). trones, de guerriers, ces prêtres, ce nuage d'encens, ce sacrifice, tout sert à donner à ce conseil un appareil imposant" (Atala, p. 49-50).

La différence de ton est manifeste : quand le voyageur brosse, avec amusement, sinon ironie, le tableau d'une assemblée de Sauvages, dans laquelle l'exotique le dispute au disparate, c'est pour ravaler bien vite cette institution indienne au rang de spectacle pittores$q u e$. Le romancier, lui, préfere parler d'un appareil imposant. Pourtant les éléments qui composent ces deux descriptions sont à peu près semblables : d'un texte à l'autre, on retrouve les chefs de guerre, les matrones, les prêtres, les jongleurs, sans compter le calumet, les plumes et les peintures corporelles. Mais leur traitement est absolument différent : tout d'abord, les verbes d'action transforment la description d'Atala en une narration postiche, et contribuent ainsi à animer la scène, le présent de narration ne faisant que renforcer l'effet d'hypotypose. La présentation des participants, qui repose sur la symétrie du nombre, l'uniformité du costume et la théâtralité de la répartition des personnages, crée un effet dramatique puissant, qui n'est pas sans évoquer l'épopée.

D'autre part, les indices de couleur locale sont systématiquement pris dans un réseau de sens qui en atténue la portée pittoresque : le calumet de paix est à demi coloré pour la guerre, les corps des guerriers ne sont plus ni tatoués ni bariolés ni peinturés, comme c'était le cas dans le récit de voyage : ils sont désormais teints de sang. Quant au hibou empaillé qui coiffe le jongleur, il ne fait qu'accentuer le caractère sacré et mystérieux du rituel en train de s'accomplir, où l'on retrouve des éléments familiers au lecteur des Incas de Marmontel, comme le feu, les gardiens du temple et le sacrifice au soleil: le conseil sauvage se transforme en un rituel pharaonique d'inspiration néo-classique.

Quelles sont les raisons d'un tel changement d'une œuvre à l'autre ? À coup sûr, les intérêts de la narration fictionnelle sont ici primordiaux : le moment est d'importance, puisqu'il introduit la discussion houleuse qui aboutira à l'arrêt de mort de Chactas. N'oublions pas en outre la préoccupation constante de Chateaubriand, conscient d'avoir

"à soutenir l'intérêt dramatique entre deux seuls personnages, pendant toute une longue peinture des mœurs, et de nombreuses descriptions de paysages $[\ldots]$ "(Préface d'Atala, p. 21). 
Quoi d'étonnant à ce qu'il cherche à dramatiser la forme de descriptions à caractère essentiellement didactique, ce que l'inventaire du Voyage en Amérique ne le contraint nullement à faire? On se contentera d'ajouter qu'à la dimension strictement narrative s'en adjoint une autre, d'ordre plus rhétorique : la dramatisation de la scène est aussi l'occasion d'un changement de registre, qui en élève la teneur en même temps que le style.

À la stylisation, à la rentabilisation dramatique des éléments descriptifs imposées par les contraintes de la fiction narrative s'adjoindrait donc une sorte de mise en forme esthétique que n'imposerait pas a priori l'écriture réputée factuelle et dépouillée du récit de voyage ${ }^{9}$. Il est facile de le vérifier au regard des analogies, en comparant, par exemple, les deux tableaux du Meschacébé ${ }^{10}$ qui sont donnés dans le Voyage en Amérique et dans l'ouverture d'Atala:

" [...] l'eau s'élève à une grande hauteur, noie ses rivages, et ne retourne point au fleuve dont elle est sortie, comme l'eau du Nil : elle reste sur la terre, ou filtre à travers le sol, sur lequel elle dépose un sédiment fertile" (Voyage en Amérique, p. 722-723).
"Par intervalles, il élève sa voix, en passant sous les monts, et répand ses eaux débordées autour des colonnades des forêts et des pyramides des tombeaux indiens; $c$ 'est le Nil des déserts" (Atala, p. 34).

Le récit de voyage se caractérise par une certaine sobriété dans l'usage de l'analogie, qui se borne à comparer a contrario le Meschacébé au Nil : il s'agit d'une comparaison à vocation didactique, qui utilise un fait avéré pour le lecteur (les crues du Nil) afin d'illustrer un phénomène a priori inconnu de lui.

Les métaphores d'Atala sont d'une tout autre portée : la dernière ( $c$ 'est le Nil des déserts) est en quelque sorte préparée par les deux précédentes (colonnades des forêts, pyramides des tombeaux indiens). Il s'agit d'une allégorie, puisqu'il existe un lien sémantique direct entre ces analogies. La fonction de cette métaphore semble certes plus décorative que didactique, mais elle pourrait être chargée d'un autre sens : elle est en effet la seule rescapée du système métaphorique développé dans un prcmicr état de ce texte, qui constitue l'un des Fragments d'une version primitive du Génie, et qui est reproduit au tome 31 des OEuvres complètes dans l'édition Pourrat

9. Dans la Préface d'Atala comme dans celle des Natchez, Chateaubriand considère explicitement la description comme un moyen de l'ornementation de l'ouvrage : "Je ne dissimule point que j'ai cherché l'extrême simplicité de fond et de style, la partie descriptive exceptée " (p. 18 - souligné par nous; cf. aussi p. 164).

10. Chateaubriand l'appelle Mississipi dans le récit de voyage. 
de $1838^{\prime \prime}$. C'est sur cette base qu'a été élaborée la description romanesque, qui a fait l'objet de remaniements successifs dans les versions postérieures à 1801 . Sans procéder à l'examen génétique de ce texte ${ }^{12}$, on constate qu'au fil des versions successives, Chateaubriand a considérablement allégé le dispositif analogique mis en place dans l'état initial, au point de faire disparaître les pesantes allégories du temps et du fleuve, qui sentaient quelque peu leur écolier. "Patient polissage de la forme sous l'effet de contraintes imposées par le goût et la raison "selon $P$. Glaudes ${ }^{13}$ ? Fidélité à la poétique chrétienne du Génie, qui ne se soucie plus d'encombrer la nature d'un appareil mythologique comparé à une " uniforme machine d'Opéra " (II, 4, 1) ? Il n'en reste pas moins que Chateaubriand persiste à placer le Nil en point d'orgue de son évocation du Meschacébé et, partant, à établir un parallèle explicite entre l'Égypte et les déserts de l'Amérique.

On s'étonnera peut-être moins de ce retour, sinon du refoulé, du moins de l'architectural et de l'antique, en songeant que l'on touche là au cœur même du projet chateaubrianien ${ }^{14}$. Le jeune disciple de Jean-Jacques qui s'embarque pour l'Amérique en 1791, loin de trouver l'homme de la pure nature qu'il attendait, fait sur place la découverte étonnante d'un peuple civilisé - et non point sauvage - mais dont la civilisation a su rester " primitive " au sens le plus noble du terme : elle ne s'est jamais dénaturée depuis les origines, sa vision de la nature est en particulier demeurée intacte. C'est peut-être là tout le sens du parallèle établi entre l'Amérique et la terre des pharaons : la civilisation indienne représente cette "substance chimiquement pure", susceptible d'être rapprochée de l'Égypte qui, selon $M$. Butor, porte en elle les " commencements de toutes les coutumes, de toutes les lois des Grecs, des Romains et des Hébreux "15. L'Indien n'est pas l'homme d'une absence de civilisation : il est celui d'une civilisation différente parce qu'originale. La métaphore et, plus généralement, la description ne se contentent donc pas d'obéir à leur vocation décorative ou dramatique : elles contiennent en germe le projet primitif des Natchez, qui consistait à recueillir par écrit les traditions et les institutions sauvages, alors à l'agonie, et qui

11. P. 34 (reproduite dans l'éd. Gallimard-Pléiade d'Atala, p. 1189-1190).

12. Cf. à ce sujet P. Glaudes, :Atala ou le désir cannibale, Paris, 1994, p. 33 et suiv.

13. Ibid., p. 35.

14. Cf. M. Butor, Chateaubriand et l'ancienne Amérique, dans Répertoire II, Paris, 1964.

15. Ibid., p. 178. 
autorise Chateaubriand à se présenter, dans la Préface du Voyage en Amérique,

" comme le dernier historien des peuples de la terre de Colomb, de ces peuples dont la race ne tardera pas à disparaître [...]" (p. 663).

Est-ce à dire pour autant que les descriptions du récit de voyage ne doivent trouver grâce aux yeux du lecteur contemporain qu'en vertu de leur seule valeur informative ou, à la rigueur, testimoniale? Ce serait faire bon marché de l'obscur, mais indéniable travail de recomposition auquel s'est livré Chateaubriand avant la publication du Voyage en Amérique en 1827, au point que l'on y a vu souvent une sorte de premier état des livres correspondants des Mémoires. On comparera, pour le vérifier, la description du cimetière indien dans Atala et dans le "Journal sans date".

L'évocation des Bocages de la mort dans Atala est, comme on sait, commandée par la fameuse allégorie de la forêt-sanctuaire, dans sa version funéraire, au point que cette description semble l'image inversée du chapitre du Génie consacré aux églises gothiques, dans lequel Chateaubriand présente les forêts comme " les premiers temples de la Divinité ", et le lieu où "les hommes ont pris [...] la première idée de l'architecture $" 16$ :

"Ce riant asile des âmes était fermé à l'orient par le pont sous lequel nous avions passé ; deux collines le bornaient au septentrion et au midi; il ne s'ouvrait qu'à l'occident, où s'élevait un grand bois de sapins. Les troncs de ces arbres, rouges marbrés de vert, montant sans branches jusqu'à leurs cimes, ressemblaient à de hautes colonnes, et formaient le péristyle de ce temple de la mort; il y régnait un bruit religieux, semblable au sourd mugissement de l'orgue sous les voûtes d'une église; mais lorsqu'on pénétrait au fond du sanctuaire, on n'entendait plus que les hymnes des oiseaux qui célébraient à la mémoire des morts une fête éternelle" (Atala, éd. citée, p. 69-70 - souligné par nous).

Il est pratiquement avéré que René n'a pas trouvé en lui-même la source de cette allégorie ${ }^{17}$. Mais elle n'en reste pas moins l'un

16. "Ces voûtes ciselées en feuillages, ces jambages, qui appuient les murs et finissent brusquement comme des troncs brisés, la fraîcheur des voûtes, les ténèbres du sanctuaire, les ailes obscures, les passages secrets, les portes abaissées, tout retrace les labyrinthes des bois dans l'église gothique; tout en fait sentir la religieuse horreur, les mystères et la divinité " (III, 1, 8).

17. M. Baridon la fait remonter à la première moitié du XVIII siècle, chez l'Anglais WARBURTON (Jardins et paysage. Existe-t-il un style anglais?, dans DixHuitieme Siècle, 18, 1986, p. 427-446). M. Regard la trouve aussi dans l'Histoire 
des piliers de la poétique du christianisme, puisqu'elle rend manifeste la présence du divin dans la nature, et l'harmonie qu'elle y fait régner. Il est donc normal qu'elle soit mise au service de l'illustration des thèses du Génie, dont se charge Atala, en particulier à ce moment du récit plus proprement consacré à la mission du père Aubry.

Or cette évocation du cimetière indien se retrouve précisément dans un passage du "Journal sans date ", que Chateaubriand avait peut-être songé à introduire, sous une forme remaniée, dans les Mémoires ${ }^{18}$. Elle précède justement une nouvelle occurrence de l'analogie arbre-colonne qui, rappelons-le, constitue l'un des termes majeurs de l'allégorie que nous venons de souligner:

\section{"Six heures}

J'avais entrevu de nouveau unc clarté et j'avais marché vers elle. Me voilà au point de lumière : triste champ plus mélancolique que les forêts qui l'environnent! Ce champ est un ancien cimetière indien. Que je me repose un instant dans cette double solitude de la mort et de la nature : est-il un asile où j'aimasse mieux dormir pour toujours?

\section{Sept heures}

Ne pouvant sortir de ces bois, nous y avons campé. La réverbération de notre bûcher s'étend au loin; éclairé en dessous par la lueur scarlatine, le feuillage paraît ensanglanté, les troncs des arbres les plus proches s'élèvent comme des colonnes de granit rouge, mais les plus distants, atteints à peine de la lumière, ressemblent, dans l'enfoncement des bois, à de pâles fantômes rangés en cercle au bord d'une nuit profonde " (Voyage en Amérique, p. 706 - souligné par nous).

La comparaison, banale en apparence, est le seul élément qui rappelle l'allégorie précédemment signalée. L'arbre-colonne ne cache plus la forêt-temple : il n'y a pas ici de cadre général à l'intérieur

des deux Indes de l'abbé Raynal (cf. son édition du Génie, Paris, Gallimard-Pléiade, 1978 , p. 1807). Mais Chateaubriand pourrait très bien s'être inspiré de Paul et Virginie : " [les] troncs mousseux et bruns [des arbres] paraissaient changés en colonnes de bronze antique " (J. Ehrard éd., Paris, Gallimard, Folio, 1984, p. 152); " [le] murmure sourd et mugissant [des palmistes et des tatamaques] ressemble au bruit lointain des orgues, et inspire une profonde mélancolie " (ibid., p. 180); "Mille bruits confus sortent de ces eaux tumultueuses, et dispersés par les vents dans la forêt, tantôt ils fuient au loin, tantôt ils se rapprochent tous à la fois, et assourdissent, comme les sons d'une cloche d'une cathédrale " (p. 196). Cf. aussi J.-P. Richard, Paysage de Chateaubriand, Paris, 1967, p. 101-102, et M. RiffaTERRE, De la structure au code: Chateaubriand et le monument imaginaire, dans La Production du texte, Paris, 1979, p. 130.

18. Cf. l'édition du centenaire procurée par M. Levaillant (Flammarion, 1948, t. I, p. 322 et suiv.). 
duquel le sens et, partant, l'interprétation pourraient s'organiser sans difficulté. Il faut poursuivre la lecture pour retrouver quelques bribes du système allégorique dans la séquence qui conclut l'évocation de cette nuit dans les forêts du Nouveau Monde:

"Est-ce les sons graves de l'orgue que j'entends, tandis que des sons plus légers errent dans les voûtes de verdure? [...] les chauves-souris accrochées aux feuilles élèvent leurs chants monotones : on croit ouir des glas continus, ou le tintement funèbre d'une cloche" (ibid., p. 707 souligné par nous).

L'allégorie est donc bien présente, mais sous la forme d'éléments épars dont le lien sémantique est beaucoup plus lâche que dans la fiction narrative. On aura en outre été sensible à la tonalité générale du passage, qui contraste avec la sérénité de l'évocation romanesque, et à l'isotopie funèbre au sein de laquelle les éléments de l'allégorie se développent : ainsi, la couleur rouge, qui caractérise la teinte des troncs d'arbres éclairés par le feu de bois et qui domine le paragraphe (lueur scarlatine), est fortement connotée par l'adjectif ensanglanté. L'impression est renforcée par la comparaison qui clôt l'alinéa, dans laquelle les arbres les plus distants sont comparés à de pâles fantômes. Dans la séquence consacrée aux bruits de la forêt, le comparant cloche est, plus nettement encore, connoté par le substantif glas, lui-même glosé par la périphrase tintement funèbre.

Pour comprendre le sens de ce traitement bien particulier de l'allégorie, il convient de resituer les passages cités dans leur contexte. Cette évocation d'une nuit dans les forêts américaines, sensiblement différente de celle qui a fait la fortune de l'Enchanteur, représente en effet la partie centrale du " Journal sans date ". Chateaubriand lui accorde d'ailleurs suffisamment d'importance pour qu'on en trouve des échos dans le reste de son œuvre ${ }^{19}$, et sans citer le texte en entier, on peut tout de même en rappeler quelques étapes, afin de lire plus exactement les passages précédemment cités. Contrairement à ce que l'on pourrait croire, la part de la narration est très limitée dans le "Journal sans date ": la description et le commentaire peuvent donc s'y donner libre cours, mais l'ensemble reste, comme on l'a vu, régi par le découpage temporel très strict du journal. La structure narrative est ainsi bien présente, mais presque totalement débarrassée du fardeau de la narration.

19. En particulier dans l'Itinéraire (dans OEuvres romanesques et voyages, éd. citée, t. II, p. 830-831), le Voyage en Italie (ibid., p. 1486) et les Mémoires (VII, 7 M. Levaillant et G. Moulinier éd., Paris, Gallimard, Pléiade, 1951, t. I, p. 242). 
Après un début marqué par l'effusion lyrique du je goûtant le bonheur de la "liberté primitive" au sein de la nature, et qui se poursuit par le récit du voyage en canot, ponctué de descriptions euphoriques, le voyageur et ses compagnons se trouvent pris au piège d'un canal trop étroit et contraints de poursuivre leur chemin à pied à travers bois. La tonalité de la séquence qui débute alors tranche sur la précédente : le récit souligne l'errance du narrateur à travers une forêt qui n'est pas sans évoquer le labyrinthe ${ }^{20}$. C'est l'occasion de mettre en place un embrayeur de tonalité ${ }^{21}$, ainsi qu'une isotopie de la transcendance ${ }^{22}$, de la chute ${ }^{23}$ et de la décomposition qui lui est liée ${ }^{24}$.

La séquence suivante est précisément celle qui est consacrée au cimetière indien, et qui renforce l'isotopie primitive par l'établissement d'un double lien sémantique: entre la mort et la lumière d'une part, entre la mort de la nature et la mort de l'homme ensuite. Puis vient celle où se met en place l'analogie entre l'arbre et la colonne: la tonalité se fait alors nettement dysphorique, avec des adjectifs comme triste, mélancolique, ensanglanté. Suit une sorte de récit où, d'un point de vue strictement événementiel, il ne se passe pratiquement rien. Pourtant, les verbes sont porteurs de sèmes ou d'aspects assez nettement orientés :

" Minuit

Le feu commence à s'éteindre, le cercle de sa lumière se rétrécit " (p. 706).

Tout indique une progression orientée vers une obscurité totale, qui dévore progressivement le cercle de lumière au centre duquel se

20. "Partout il faut franchir des arbres abattus [...]. Je cherche en vain une issue dans ces solitudes; trompé par un jour plus vif, j'avance à travers les herbes $[\ldots]$ mais je n'arrive qu'à une clairière $[\ldots]$ ". D'où la résolution prise à sept heures : "Ne pouvant sortir de ces bois, nous y avons campé " (p. 706). À noter la présence d'illusions optiques, qui prennent l'allure de prestiges destinés à décevoir le voyageur (le jour plus vif, la clarté, qui ne révèlent qu'une clairière sans issue ou un cimetière indien) ou à le tromper (les troncs d'arbres qui " se succèdent les uns les autres, et qui semblent se serrer en s'éloignant " ou qui ressemblent à de pâles fantômes) (ibid.).

21. " [...] une demi-lumière changeante et mobile, qui donne aux objets une grandeur fantastique" (p. 705-706 - souligné par nous).

22. "Qui dira le sentiment qu'on éprouve en entrant dans ces forêts aussi vieilles que le monde, et qui seules donnent une idée de la création, telle qu'elle sortit des mains de Dieu? [...] l'idée de l'infini se présente à moi " (ibid.).

23. "le jour tombant d'en haut ", " des arbres abattus ", "quelques pins tombés" (ibid.).

24. "l'épais humus composé des débris des végétaux" (p. 706). 
trouve placé le voyageur. À compter de ce moment d'ailleurs, la perception visuelle disparaît du texte, et se voit remplacée par des impressions uniquement sonores. Tous ces faits, stylistiques ou non (tonalité dysphorique et isotopie funèbre, errance dans le labyrinthe et orientation des événements, illusions sensorielles et sensation de l'infini), semblent donc se conjuguer pour montrer que la progression chronologique linéaire à laquelle obéit le texte se se déroule en contrepoint d'une autre, d'ordre imaginaire et symbolique, dont cette séquence constitue précisément une étape essentielle.

À la nuit complète s'ajoute bientôt le silence total :

"J'écoute : un calme formidable pèse sur ces forêts; on dirait que des silences succèdent à d'autres silences. Je cherche vainement à entendre dans un tombeau universel quelque bruit qui décèle la vie" (p. 706-707).

Cette étape est marquée par l'apparition d'un nouvel indicateur de tonalité : alors que l'adjectif fantastique semblait renvoyer au monde de l'illusion et de la fantaisie - représentée par les illusions d'optique, l'adjectif formidable paraît indiquer au contraire le voisinage du sacré, représenté par ce tombeau universel dans lequel l'obscurité, le silence et la mort sont étroitement associés. L'analogie arbre-colonne retrouve, dans ce contexte, le sens funèbre qu'elle avait dans Atala: on associera sans grand risque d'extrapolation le granit et le mausolée. Elle donne du même coup, selon un procédé classique de l'écriture chatcaubrianienne, une consistance architecturale à ce tombeau imaginaire. Mais la dimension chrétienne et presque euphorique qui marquait l'allégorie romanesque est ici absente : elle laisse place à une vision essentiellement mortuaire.

Il n'est pas inintéressant de souligner d'autre part que Chateaubriand utilise pratiquement la même structure de phrase que lorsqu'il narrait son errance dans le labyrinthe de la forêt (Je cherche en vain une issue dans ces solitudes) : la démarche au sein de ce simulacre de la mort est peut-être la même, mais alors que le cheminement réel se soldait par un échec, celui-ci, d'ordre symbolique, semble plus positif, malgré les apparences, puisque la tournure de la phrase lui permet de s'achever sur la vie. Déjà l'évocation du silence absolu qui ouvre l'épisode (on dirait que des silences succèdent à d'autres silences) suggérait une organisation du vide qui vaut davantage pour sa formulation que pour son contenu : cette forme donnée au silence est déjà le témoignage d'une conscience créatrice qui cherche, selon le mot de J.-P. Richard, à "éveiller la vacance de l'espace ", moins pour la nier peut-être que pour 
l'apprivoiser ${ }^{25}$. De fait, le bruit ne reparaît que dans une structure purifiée de tout parasite:

"D'où vient ce soupir? d'un de mes compagnons : il se plaint, bien qu'il sommeille. Tu vis, donc tu souffres : voilà l'homme" (ibid.).

L'étrange aphorisme que le narrateur tire de cette anecdote est beaucoup moins anodin qu'il n'y paraît : s'il renvoie à l'évidence à la très chateaubrianienne philosophie du "malheur d'être né ", sa formulation mi-cartésienne mi-évangélique ne laisse pas d'étonner et d'offrir matière à conjectures. Si la vie est effectivement placée, par le biais de cet ecce homo, sous le signe de la souffrance christique, comment ne pas rapprocher le "dépouillement du monde " mis en scène dans ce passage et l'expérience du Discours de la méthode ou des Méditations? Faut-il voir dans ce " partage de minuit "l'émergence d'une forme de cogito chateaubrianien, moins métaphysique qu'existentiel, et comme mis à distance par son attribution à un étrange allocutaire, dont on ne saurait dire s'il est le dormeur gémissant ou une personne abstraite? La question, à défaut d'être résolue, mérite au moins d'être posée...

La séquence suivante associe contradictoirement le mouvement et l'immobilité, le bruit et le silence, la vie et la mort, qui semblent s'affronter en un ultime combat :

\section{“Minuit et demi}

Le repos continue; mais l'arbre décrépit se rompt: il tombe. Les forêts mugissent ; mille voix s'élèvent. Bientôt les bruits s'affaiblissent; ils meurent dans des lointains presque imaginaires : le silence envahit de nouveau le désert" (p. 707).

Tout en prolongeant l'isotopie mortuaire, cette séquence marque l'émergence de la dimension sonore sous une forme narrativisée qui relance l'intérêt. La seconde phrase introduit un sentiment, non plus visuel, mais sonore de l'infini : si l'on reprend l'analyse de J.-P. Richard, le narrateur se fait annoncer la profondeur en suivant l'onde sonore qui s'y perd et " ressent l'infinité de l'étendue à travers l'échec du mouvement qui prétendait la lui faire parcourir " ${ }^{26}$. Du même coup, le silence prend, comme précédemment, une véritable consistance, puisqu'il envahit de nouveau le désert.

25. Cf. J.-P. RICHARD, ouvr. cité, p. 51-52.

26. Ibid., p. 81 . 
L'arrivée du vent dans la dernière séquence nocturne, mise en scène par le présentatif, marque, en dépit d'une comparaison modalisante, le triomphe de la vie et du mouvement :

\section{"Une heure du matin}

Voici le vent; il court sur la cime des arbres; il les secoue en passant sur ma tête. Maintenant c'est comme le flot de la mer qui se brise tristement sur le rivage.

Les bruits ont réveillé les bruits. La forêt est toute harmonie. Est-ce les sons graves de l'orgue que j'entends, tandis que des sons plus légers errent dans les voûtes de verdure ? Un court silence succède; la musique aérienne recommence; partout de douces plaintes, des murmures qui renferment en eux-mêmes d'autres murmures; chaque feuille parle un différent langage, chaque brin d'herbe rend une note particulière " (ibid.).

Les verbes d'action se succèdent et, associés qu'ils sont à des sujets inanimés, semblent anthropomorphiser la nature. Si la figure de l'infini se maintient, elle paraît apprivoisée. Les murmures de la forêt sont décrits, non comme un chaos, mais comme une " harmonie ", une "musique aérienne ", dont le narrateur est en mesure de distinguer chaque partie : on attendait un pandémonium, c'est une Pentecôte de la nature à laquelle le vent, en " passant sur [s]a tête ", invite le je acteur et narrateur. Mais n'est-ce pas justement au cours de cette nuit qu'aux dires des Mémoires apparut à René " une muse inconnue "?

" $[\ldots]$ je recueillis quelques-uns de ses accents; je les marquai sur mon livre, à la clarté des étoiles, comme un musicien vulgaire écrirait des notes que lui dicterait quelque grand maître des harmonies"(VII, 7 - éd. citée, t. I, p. 242).

On comprend mieux que l'allégorie de la forêt-sanctuaire se volatilise en quelque sorte dans ces lignes : cet éparpillement même montre que sa portée dépasse ici largement le sème architectural ou apologétique qui lui était attribué dans Atala.

Il est sûr que la fin de la séquence rappelle à la réalité du " tombeau universel " en préparant la sentence ultime :

"Une voix extraordinaire retentit : c'est celle de cette grenouille qui imite les mugissements du taureau. De toutes les parties de la forêt, les chauves-souris accrochées aux feuilles élèvent leurs chants monotones : on croit ouir des glas continus, ou le tintement funèbre d'une cloche. Tout nous ramène à quelque idée de la mort, parce que cette idée est au fond de la vie "(ibid.). 
Mais celle-ci est-elle autre chose que la reformulation, abstraite et raisonnée, du message qui était déjà présent au début du texte, dans l'évocation " des arbres abattus sur lesquels s'élèvent d'autres générations d'arbres " (p. 706) ? Imaginaire et symbolique, cette traversée de la mort a simplement permis au je de prendre conscience de ce message.

On a interprété le "Journal sans date " comme l'échec de "la tentative de se familiariser avec cette nature indomptable ":

"L'homme en fuite devant la civilisation de l'Ancien Monde ne trouve pas de refuge dans la nature sauvage, bien au contraire, elle s'oppose à lui avec une hostilité sublime qui lui montre son impuissance et sa fragilité et le renvoie à une idée religieuse " 27 .

C'est peut-être négliger un peu la conclusion de ce passage : si les explorateurs renoncent effectivement à poursuivre leur randonnée dans un milieu auquel, contrairement aux Indiens, ils ne sont pas adaptés, ce renoncement est plutôt bien accepté. Les gélinottes offrent des repas savoureux, la nuit est paisible, les Indiens rencontrés peu après sont hospitaliers et prêts à fraterniser. On retrouve l'euphorie initiale, qui tranche nettement sur le ton de la séquence centrale ${ }^{28}$.

Mais voir dans le "Journal sans date " un simple constat d'échec, c'est surtout faire un peu trop bon marché du développement très construit que l'on a tenté de mettre en lumière : qu'est-ce que ce " Journal sans date ", sinon la mise en scène d'une initiation? Tous les éléments sont en place : errance dans le labyrinthe, leurres, sentiment d'effroi, traversée symbolique de la mort, révélation au néophyte purifié d'un message qu'il n'avait pas su percevoir auparavant, et retrouvailles finales avec le monde. On a eu quelques aperçus de la complexité de la révélation : sentiment douloureux de la fluidité de l'existence, liée à l'omniprésence de la mort, inspiration liée à la nature. On est là au cœur palpitant de l'imaginaire chateaubrianien et, dans la suite de l'œuvre, cet épisode servira bien souvent de point-origine au narratcur pour mesurer l'évolution de son moi et éprouver ainsi son épaisseur temporelle ${ }^{29}$.

Le Voyage en Amérique est donc - au moins partiellement - porteur d'une démarche symbolique dans laquelle la description joue

27. T. Michaelis, De Bernardin de Saint-Pierre à Chateaubriand : un tournant dans l'histoire du voyage en littérature, dans Le Journal de voyage et Stendhal. Actes du colloque de Grenoble (V. del Litto et E. Kanceff éd.), Genève, 1986, p. 81.

28. P. 707-709.

29. Cf. supra, note 19 . 
un rôle déterminant. Mais ce rôle est fort différent de celui qu'elle occupe dans le récit de fiction correspondant : si, par exemple, la métaphore de l'arbre-colonne reprend des véhicules analogues à ceux qu'elle utilisait dans Atala, sa portée dépasse largement celle de la forêt-sanctuaire, en voie de banalisation. Au contact du développement linéaire, sinon narratif, du journal, elle s'enrichit de nouveaux sèmes : infini spatial (lignes de fuite) et temporel (succession des générations), thèmes mortuaire et religieux vus sous un jour moins architectural que métaphysique. Le lien entre la nature et l'homme par la communauté de la vie, de la mort et du temps se fait beaucoup plus explicite que dans l'extrait romanesque, et se charge d'un contenu existentiel qui en était absent.

Le plus important reste toutefois la contribution de la description à cette démarche évolutive : en l'absence d'un fort schéma et même d'un vrai contexte - narratif, la juxtaposition des séquences descriptives épouse les méandres de la conscience du $j e$ acteur aux prises avec son imaginaire, et contribue à la mise en scène qu'en donne le $j$ e narrateur et auteur. On a le sentiment très net qu'en pervertissant la forme du journal intime, c'est la description qui guide cette maïeutique, psychologique et stylistique, pour aboutir à l'un des archétypes de l'univers intime de Chateaubriand: le narrateur des Mémoires saura s'en souvenir.

Il n'est donc guère étonnant qu'à terme René, le descripteur, ait préféré renoncer aux contraintes de la fiction romanesque pour se couler dans le moule infiniment plus souple de l'écriture autobiographique - dont ce récit de voyage, avant même l'Itinéraire, donne déjà une idée. Peu importe alors s'il a effectivement séjourné dans ces forêts américaines : la vérité qui nous est révélée est d'un autre ordre que celui qui associe fiction et mensonge. Et - une fois n'est pas coutume - on laissera le mot de la fin à Sainte-Beuve, lequel, peu soucieux des " inadvertances de souvenir " qui émaillent le Voyage en Amérique, en loue au contraire la sincérité :

" [...] dans ce journal sans date, dans ces forêts sans nom, en descendant ce fleuve qu'il ne nomme pas davantage, c'est là qu'il est en pleine vérité, qu'il abonde et qu'il nage en plein sentiment de la nature américaine. Et qui donc nous l'a révélé le premier comme lui ? " 30

Alain GuYOT.

Université de Paris III.

30. Chateaubriand et son groupe littíraire sous l'Empire, Paris, Garnier, 1861, t. I, p. 127 . 